\title{
Paraoxonase 1 Genetic Polymorphisms in a Mixed Ancestry African Population
}

\author{
M. Macharia, ${ }^{1}$ A. P. Kengne, ${ }^{2}$ D. M. Blackhurst, ${ }^{3}$ R. T. Erasmus, ${ }^{1}$ and T. E. Matsha ${ }^{4}$ \\ ${ }^{1}$ Division of Chemical Pathology, Faculty of Health Sciences, National Health Laboratory Service (NHLS) and \\ University of Stellenbosch, Cape Town 7505, South Africa \\ ${ }^{2}$ Non-Communicable Diseases Research Unit, South African Medical Research Council, University of Cape Town, \\ Cape Town 7505, South Africa \\ ${ }^{3}$ Division of Chemical Pathology, University of Cape Town, Cape Town 8000, South Africa \\ ${ }^{4}$ Department of Biomedical Sciences, Faculty of Health and Wellness Science, Cape Peninsula University of Technology, \\ P.O. Box 1906, Bellville, Cape Town 7530, South Africa
}

Correspondence should be addressed to T. E. Matsha; matshat@cput.ac.za

Received 9 July 2014; Revised 20 October 2014; Accepted 21 October 2014; Published 16 November 2014

Academic Editor: Vinod K. Mishra

Copyright (C) 2014 M. Macharia et al. This is an open access article distributed under the Creative Commons Attribution License, which permits unrestricted use, distribution, and reproduction in any medium, provided the original work is properly cited.

\begin{abstract}
Paraoxonase 1 (PON1) activity is markedly influenced by coding polymorphisms, Q/R at position 192 and M/L at position 55 of the PON1 gene. We investigated the frequencies of these polymorphisms and their effects on PON1 and antioxidant activities in 844 South African mixed ancestry individuals. Genotyping was done using allele-specific TaqMan technology, PON1 activities were measured using paraoxon and phenylacetate, oxidative status was determined by measuring the antioxidant activities of ferric reducing antioxidant power and trolox equivalent antioxidant capacity, and lipid peroxidation markers included malondialdehyde and oxidized LDL. The frequencies of Q192R and L55M were $47.6 \%$ and $28.8 \%$, respectively, and the most common corresponding alleles were 192R (60.4\%) and 55M (82.6\%). The Q192 was significantly associated with 5.8 units' increase in PON1 concentration and 15.4 units' decrease in PONase activity after adjustment for age, sex, BMI, and diabetes, with suggestion of differential effects by diabetes status. The PON1 L55 variant was associated with none of the measured indices. In conclusion, we have shown that the Q192R polymorphism is a determinant of both PON1 concentration and activity and this association appeared to be enhanced in subjects with diabetes.
\end{abstract}

\section{Introduction}

Paraoxonase 1 (PON1) is a calcium dependent esterase synthesized in the liver and widely distributed in tissues including liver, kidney, intestine, and serum, where it associates with high-density lipoprotein (HDL). The enzyme has a dual physiological function in humans. First, it catalyzes the breakdown of various toxic organophosphate $(\mathrm{OP})$ pesticides and nerve gases, including paraoxon, diazoxon, sarin, and soman $[1,2]$, which are potent acetylcholinesterase (AChE) inhibitors. Secondly, PON1 is increasingly acknowledged as an atheroprotective enzyme due to its in vitro ability to inhibit oxidative modifications of LDL [3], HDL [4], macrophages [5], atherosclerotic lesions [6], and augment cholesterol efflux from macrophages [7]. In addition, PON1 lowers inflammatory responses in the arterial wall by destroying biologically active lipids in mildly oxidized LDL [8], impairing the differentiation of monocytes to macrophages [9], and decreasing monocyte chemotaxis and adhesion to endothelial cells [10]. Decreased PON1 activities have been reported in diseases with accelerated atherogenesis including diabetes and familial hypercholesterolemia [11-13].

The activity of the enzyme is markedly influenced by polymorphisms on the coding and promoter regions of the PON1 gene. The coding polymorphisms are $\mathrm{Q} / \mathrm{R}$ at position 192 and M/L at position 55 which result in isozymes differing greatly in their activity toward various substrates $[2,14,15]$. The R isoform hydrolyzes paraoxon faster than the Q isoform, 
whereas diazoxon, soman, and sarin are hydrolyzed at a higher rate by the $\mathrm{Q}$ than $\mathrm{R}$ isoform [2]. In contrast, the $\mathrm{R}$ isoform is less effective at hydrolyzing lipid peroxides than the $\mathrm{Q}$ isoform [2]. The $\mathrm{M}$ and $\mathrm{L}$ alleles are associated with lower and higher serum PON1 concentrations, respectively [16]. The distribution of the Q192R and L55M polymorphisms widely varies worldwide. For example, the frequency of the PON1 Q192 allele has a high frequency in Caucasians (0.70) [17, 18 ], but a considerably lower frequency in Mexicans (0.48) [19] and African-Americans (0.34) [18]. The PON1 L55 allele predominates in nearly all populations but variations still exist, for example, between Taiwanese (0.97) [20], Gabonese (0.695) [21], Turkish (0.39) [22], and Iranians (0.59) [23].

This study was undertaken to investigate the frequencies of PON1 Q192R and L55M polymorphisms and their possible relationship with PON1 activity and indices oxidative status (ferric reducing antioxidant power, trolox equivalent antioxidant capacity, malondialdehyde, and oxidized LDL). Herein, we investigated the mixed ancestry population from South Africa that has been shown to have one of the highest prevalence of type 2 diabetes in South Africa and sub-Saharan Africa at large [24].

\section{Materials and Methods}

2.1. Study Setting and Population. Details of the study including survey design and procedures have been described elsewhere [24, 25]. Study participants were members of a cohort study conducted in a mixed ancestry township (Bellville South) which is located within the Northern suburbs of Cape Town, Western Cape, South Africa. The mixed ancestry population of South Africa, sometimes referred to as "coloured," is of mixed genetic origin with contributions from Europeans, South Asians, Indonesians, and a population genetically close to the isiXhosa sub-Saharan Bantu [26]. The study was approved by the research ethics committees of Stellenbosch University (reference number: N10/04/118) and the Cape Peninsula University of Technology (CPUT/HW-REC 2010/H017) and was conducted according to the Code of Ethics of the World Medical Association (Declaration of Helsinki). All participants signed written informed consent after all the procedures were fully explained in the language of their choice. All participants received a standardized interview and physical examination during which blood pressure was measured according to the World Health Organisation (WHO) guidelines [27] using a semiautomated digital blood pressure monitor (Rossmax PA, USA) on the right arm in a sitting position. Anthropometric measurements were performed three times and their average used for analysis: weight $(\mathrm{kg})$, height $(\mathrm{cm})$, and waist $(\mathrm{cm})$ and hip $(\mathrm{cm})$ circumferences. Participants with no history of doctor diagnosed diabetes mellitus underwent a $75 \mathrm{~g}$ oral glucose tolerance test (OGTT) as recommended by the WHO [28]. Further, the following biochemical parameters were determined on the Cobas 6000 Clinical Chemistry instrument (Roche Diagnostics, Germany): fasting plasma glucose, insulin, total cholesterol (TC), high density lipoprotein cholesterol (HDL-c), triglycerides (TG),
C-reactive protein (CRP), $\gamma$-glutamyltransferase (GGT), and glycated haemoglobin (HbAlc) certified by National Glycohaemoglobin Standardisation Programme (NGSP). Low density lipoprotein cholesterol (LDL-c) was calculated using Friedewald's formula [29]. Serum cotinine was measured by chemiluminescent assay (Immulite 1000, Siemens).

2.2. Total Antioxidant Capacity. The total antioxidant capacity in plasma samples was assessed using the ferric reducing antioxidant power (FRAP) and trolox equivalent antioxidant capacity (TEAC) assays. FRAP was done according to the method of Benzie and Strain [30]. Briefly, plasma samples were mixed with FRAP reagent, incubated for $30 \mathrm{~min}$ at $37^{\circ} \mathrm{C}$, and the absorbance at $593 \mathrm{~nm}$ was recorded using a spectrophotometer (Spectramax plus384 Molecular devices, USA). The TEAC assay was according to Re et al. [31] and is based on monitoring (at $734 \mathrm{~nm}$ ) the oxidation of 2,2' -azinobis-(3-ethylbenzothiazoline-6-sulfonic acid) radical (ABTS) cation formed by reacting ABTS and potassium persulfate. Distilled water was used instead of PBS to dilute the $\mathrm{ABTS}^{+}$radical solution.

2.3. Paraoxonase Activity. Paraoxonase (PONase) and arylesterase (AREase) activities were measured using paraoxon and phenylacetate (Sigma Aldrich, SA) as substrates, respectively. PONase activity was measured using the method of Richter and Furlong [32] from the initial velocity of p-nitrophenol production at $37^{\circ} \mathrm{C}$ and the increased absorbance at $405 \mathrm{~nm}$ was monitored on a spectrophotometer (Spectramax plus384, Molecular devices, USA). Each serum sample was incubated with $5 \mathrm{mmol} / \mathrm{L}$ eserine (Sigma Aldrich, SA) for 15 minutes at room temperature to inhibit serum cholinesterase activity which is usually elevated in diabetes and would otherwise interfere with the determination of paraoxonase activity in serum from diabetic individuals. PON-1 activity of $1 \mathrm{U} / \mathrm{L}$ was defined as $1 \mu \mathrm{mol}$ of p-nitrophenol hydrolyzed per minute. A slightly modified method of Browne et al. [33] was used to measure AREase activity. The working reagent consisted of $20 \mathrm{mmol} / \mathrm{L}$ Tris$\mathrm{HCl}, 4 \mathrm{mmol} / \mathrm{L}$ phenyl acetate, $\mathrm{pH} 8.0$, with $1.0 \mathrm{mmol} / \mathrm{L}$ $\mathrm{CaCl}_{2}$ (Sigma Aldrich, SA). The reaction was initiated by adding $5 \mu \mathrm{L}$ of 40 -fold tris-diluted samples to $345 \mu \mathrm{L}$ of the working reagent at $25^{\circ} \mathrm{C}$. The change in absorbance at $270 \mathrm{~nm}$ was recorded for 60 minutes after a 20-second lag time on a Spectramax plus384 spectrophotometer. The activity, expressed as $\mathrm{kU} / \mathrm{L}$, was based on the molar absorptivity (1310) of phenol at $270 \mathrm{~nm}$. In both assays, the rates used to generate the data points were derived from the linear portions of the rate versus time plots.

2.4. Lipid Peroxidation. Plasma MDA and ox-LDL were used as markers of lipid peroxidation (LPO). The method of Jentzsch et al. [34] was used to estimate the thiobarbituric acid reactive substances (TBARS) which reflect the production of MDA. Plasma ox-LDLs were measured using a quantitative sandwich ELISA kit (Cellbiolabs, San Diego, California). 
TABle 1: Genotype distributions, minor allele frequencies, and unadjusted $P$ values for comparing genotype distributions according to diabetes status, and additive allelic effects between diabetes groups.

\begin{tabular}{lcccccc}
\hline & Without diabetes & Diabetes & $P$ & Overall & Men & Women \\
\hline$N$ & 606 & 238 & & 844 & 208 & 636 \\
\hline QQ192, $n(\%)$ & $97(16.0)$ & $36(15.1)$ & 0.028 & $133(15.8)$ & $32(15.4)$ & $101(15.9)$ \\
Q192R, $n(\%)$ & $272(44.9)$ & $130(54.6)$ & & $402(47.6)$ & $104(50.0)$ & $298(46.9)$ \\
192RR, $n(\%)$ & $237(39.1)$ & $72(30.3)$ & & $309(36.6)$ & $72(34.6)$ & $237(37.3)$ \\
Q, $n(\%)$ & $466(38.4)$ & $202(42.4)$ & & $668(39.6)$ & $168(40.4)$ & $500(39.3)$ \\
R, $n(\%)$ & $746(61.6)$ & $274(57.6)$ & & $1020(60.4)$ & $248(59.6)$ & $772(60.7)$ \\
HWE $(P$ value $)$ & 0.199 & 0.085 & & 0.943 & 0.666 & 0.678 \\
& & & & & \\
$55 M M, n(\%)$ & $420(69.3)$ & $156(65.6)$ & 0.168 & $576(68.3)$ & $152(73.1)$ & $424(66.7)$ \\
L55M, $n(\%)$ & $172(28.4)$ & $71(29.8)$ & & $243(28.8)$ & $54(26.0)$ & $189(29.7)$ \\
LL55, $n(\%)$ & $14(2.3)$ & $11(4.6)$ & & $25(3.0)$ & $2(1.0)$ & $23(3.62)$ \\
L, $n(\%)$ & $200(16.5)$ & $93(19.5)$ & & $293(17.4)$ & $58(13.9)$ & $235(18.5)$ \\
M, $n(\%)$ & $1012(83.5)$ & $383(80.5)$ & & $1395(82.6)$ & $358(86.1)$ & $1037(81.5)$ \\
HWE $(P$ value $)$ & 0.556 & 0.412 & & $>0.999$ & 0.383 & 0.694 \\
\hline
\end{tabular}

HWE: Hardy-Weinberg Equilibrium (HWE $P$ values are from exact tests).

2.5. Genotyping. DNA was extracted from whole blood using the salting-out method of Miller et al. [35]. Conventional polymerase chain reaction (PCR) followed by direct DNA sequencing was performed for detection of the wild type, heterozygous, and homozygous genotypes of PON1 single nucleotide polymorphisms (SNPs), Q192R (rs662, A>G), and L55M (rs854560, T>A). These internal control samples were subsequently used for analytical validation of high throughput genotyping performed on DNA samples extracted from the study participants, using the Applied Biosystems (ABI) TaqMan SNP Genotyping Assays on the ABI Prism 7900HT platform (Applied Biosystems, USA).

2.6. Definitions. Body mass index (BMI) was calculated as weight per square meter $\left(\mathrm{kg} / \mathrm{m}^{2}\right)$ and waist-hip-ratio (WHR) as waist/hip circumferences $(\mathrm{cm})$. Type 2 diabetes status was based on a history of doctor-diagnosis, a fasting plasma glucose $\geq 7.0 \mathrm{mmol} / \mathrm{L}$, and/or a 2 -hour post-OGTT plasma glucose $\geq 11.1 \mathrm{mmol} / \mathrm{L}$. The homeostatic model assessment of insulin resistance (HOMA-IR) was calculated according to the following formula: HOMA-IR = [fasting insulin concentration $(\mathrm{mIU} / \mathrm{L}) \times$ fasting plasma glucose $(\mathrm{mmol} / \mathrm{L})] / 22.5$; while functional $\beta$-cells (HOMA-B\%) were estimated using the formula: $20 \times$ fasting insulin $(\mu \mathrm{IU} / \mathrm{mL}) /$ fasting glucose $(\mathrm{mmol} / \mathrm{mL})-3.5$. The quantitative insulin-sensitivity check index (QUICKI) as $1 /[\log ($ fasting insulin $(\mu \mathrm{U} / \mathrm{mL})) \times$ $\log ($ fasting glucose $(\mathrm{mg} / \mathrm{dL}))]$.

2.7. Statistical Methods. Of the 946 participants who took part in the survey, 941 consented for genetic studies. Among the latter, 103 were excluded for missing data on the genetic variables. Oxidative status profile was assessed in 491 subjects, but 121 were also excluded on account of missing consent or fully matching biochemical and genetic data. Therefore, 844 and 370 subjects had valid data for the overall genetic and genetic-oxidative stress analyses, respectively. General characteristics of the study participants are summarized as count and percentage for dichotomous traits, mean and standard deviation (SD), or median and 25th-75th percentiles for quantitative traits. Traits were log-transformed to approximate normality, where necessary, prior to analysis. SNPs were tested for departure from Hardy-Weinberg Equilibrium (HWE) expectation via a chi square goodness of fit test. Linkage disequilibrium (LD) was estimated using the $\mathrm{D}^{\prime}$ statistic. The chi square analysis of the variance (ANOVA) and Kruskal Wallis test were used to compare baseline characteristics across allele's distribution. The interaction between SNPs was assessed through robust linear regression models, assuming additive models for the SNPs. Results corresponding to $P$ values below $5 \%$ are described as significant. We did not adjust for multiple testing. All analyses used the statistical package R (version 3.0.0 [2013-04-03], The R Foundation for statistical computing, Vienna, Austria).

\section{Results}

3.1. Distribution of PON1 Polymorphisms. Of the 844 subjects (men 208, 24.6\%) 238 (28.2\%) had diabetes, 478 (56.6\%) were hypertensive, and the average age was 56.6 (15.5) years. Genotype and allele frequencies for the L55M and Q192R polymorphisms in the overall cohort as well as across genders and diabetes status are summarized in Table 1. The frequencies of Q192R and L55M were $47.6 \%$ and $28.8 \%$, respectively, and the most common corresponding alleles were 192R (60.4\%) and $55 \mathrm{M}(82.6 \%)$. Frequencies for both polymorphisms were not different between major subgroups, except for the Q192R genotype which differed significantly according to diabetes status $(P=0.028)$. Observed and expected frequencies for both polymorphisms were in HardyWeinberg equilibrium overall and within major subgroups 
(all $P \geq 0.085$ ). The linkage between the two polymorphisms was moderate in the overall sample $\left(\mathrm{D}^{\prime}=0.470, P<0.0001\right)$ but comparatively weaker in men $\left(\mathrm{D}^{\prime}=0.199, P=0.0001\right)$, women $\left(\mathrm{D}^{\prime}=0.290, P<0.0001\right)$, and in participants with $\left(\mathrm{D}^{\prime}=0.219, P<0.0001\right)$ or without diabetes $\left(\mathrm{D}^{\prime}=0.283\right.$, $P<0.0001)$.

3.2. Baseline Profile Overall and Across PON1 Genotypes. The distribution of the baseline characteristics was not different within the various PON1 genotypes with respect to age, sex, adiposity, lipid profile prevalence of hypertension, and insulin resistance (Table 2$)$. This, however, was not the case for prevalent diabetes $(P=0.028)$ and systolic blood pressure levels $(P=0.04)$ across $\mathrm{Q} 192 \mathrm{R}$ polymorphism, and fasting plasma glucose $(P=0.019), 2 \mathrm{hr}$ glucose $(P=0.038)$, and HbAlc $(P=0.004)$ across L55M polymorphism (Table 2$)$.

\subsection{PON1 and Oxidative Status Profile Across Genotypes.} Table 3 shows the distribution of indices of PON1 and antioxidant activities across the genotypes. PON1 $(P=$ $0.015)$, PONase $(P<0.0001)$, Ox-LDL $(P=0.029)$, TBARS $(P=0.006)$, and to some extent TEAC $(P=0.053)$ were significantly different across genotype of PON1 Q192R polymorphism, whereas fasting glucose $(P=0.019)$, 2-hour glucose $(P=0.038)$, and HbAlc $(P=0.0004)$ varied across PON1 L55M genotypes.

Table 4 shows the results from robust linear regression analyses for the prediction of PON1 and antioxidant status indices by the two PON1 variants. The PON1 Q192 polymorphism was significantly associated with PON1 concentration and PONase activity resulting in unit increases of 6.8 and decreases of 18.3, respectively. The association remained significant when the models were expanded stepwise to include age, sex, BMI, and diabetes with only modest attenuation of the effect size. However, when the interaction term of diabetes and Q192 was added to multivariable models, the main effect of the polymorphism remained significant for the prediction of PONase but was substantially attenuated for PON1 concentration with a borderline association $(\beta=$ 3.79, $P=0.08$ ). Furthermore, the effect of the interaction term diabetes* Q192 was significant for the prediction of PON1 concentration (interaction $P=0.007$ ), but not for PONase, suggesting that the effect of the variant on PON1 concentration was more important in participants with diabetes (Table 4). Alone or with the other covariates in the models, the PON1 L55 was not significantly associated with any of the measured indices. However, there was a suggestion of a significant interaction by diabetes status in the effect of PON1 L55 on ox-LDL levels (interaction $P=0.013$ ), with suggestion of a positive effect on ox-LDL levels in participants with diabetes and a negative or no effect in participants without diabetes $(\beta=-395.49, P=0.075)$ for the main effect of the variant in the multivariable model containing the interaction term gene* diabetes. In the polymorphisms only model (with and without their interaction term), the effects of variants on PON1 concentration and PONase remained significant for PON1 Q192 and nonsignificant for PON1 L55.
Effects on other analytes remained unchanged with always no evidence of Q192* L55 polymorphic interaction (Table 4).

\section{Discussion}

Paraoxonase 1 polymorphisms Q192R and L55M have been reported to explain over $90 \%$ of total phenotypic variance in PON1 activity using several substrates of the enzyme [36]. In this study, we used paraoxon and phenylacetate substrates to characterize the influence of both Q192R and L55M PON1 polymorphisms on PON1 concentrations and enzyme activity in a mixed ancestry population from South Africa. Only the Q192R appeared to be functional in this population as it was associated with both PON1 concentration and the paraoxonase activity. We observed that the presence of Q192 was associated with $15.4 \mathrm{U} / \mathrm{L}$ decrease and $5.8 \mu \mathrm{g} / \mathrm{mL}$ increase in PON1 activity and concentration after accounting for the effects of age, sex, BMI, and diabetes. There was indication that the effect of the variant on PON1 concentration was more important in participants with diabetes. In parallel, we report PON1 QQ192 to be associated markers of oxidative stress (ox-LDL and TBARS) and total antioxidants (TEAC) only in cross-genotype comparison, but not in linear regression (irrespective of the level of adjustment), possibly suggesting the absence of a relationship, the nonlinearity of the association if any, or the inadequacy of the log-additive model to approximate such an association.

PON1 activity can be measured using different substrates and reports have shown that the effect of PON1 polymorphisms varies according to the substrate used $[2,14,15]$. Among three of the commonly used substrates (paraoxon, phenyl acetate, and dihydrocoumarin), the most pronounced genotype effects were for PON1 paraoxon [36]. Previous studies have associated the $\mathrm{R}$ allele with higher risk and/or incidence of atherosclerotic heart disease in various populations such as Indians [37], Japanese [38], and Dutch [39]. On the other hand, Bhattacharyya et al. [40] conducted a prospective study of 1399 patients and reported higher serum levels of PON1 activity, lower systemic indices of systemic oxidative stress, and corresponding reductions in both prevalent coronary artery disease and prospective cardiac events in PON1 192RR carriers. Similarly, the findings of the present study appear to suggest a decreased atherosclerotic risk in subjects with PON1 192R since the presence in the PON1 Q192 was significantly associated with reduction of PON1 activity which in turn has been associated with the development of CVD [41, 42]. Furthermore, PON1 QQ192 genotype was associated with increased PON1 concentration especially in subjects with diabetes. Our results could perhaps be explained by the different effect of the genotypes on HDLbound PON1 since PON1 has to be bound to HDL to perform its antiatherosclerosis function [4]. The Q192 alloenzyme binds to the HDL particle with 3-fold lower affinity than the R192 alloenzyme [43], but the HDL-bound QQ192 PON1 has been shown to be more effective at protecting the oxidation of LDL $[2,44]$. Furthermore, it has been shown that increased HDL-bound PON1 content does not alter the HDL composition or properties but protects it from lipid 


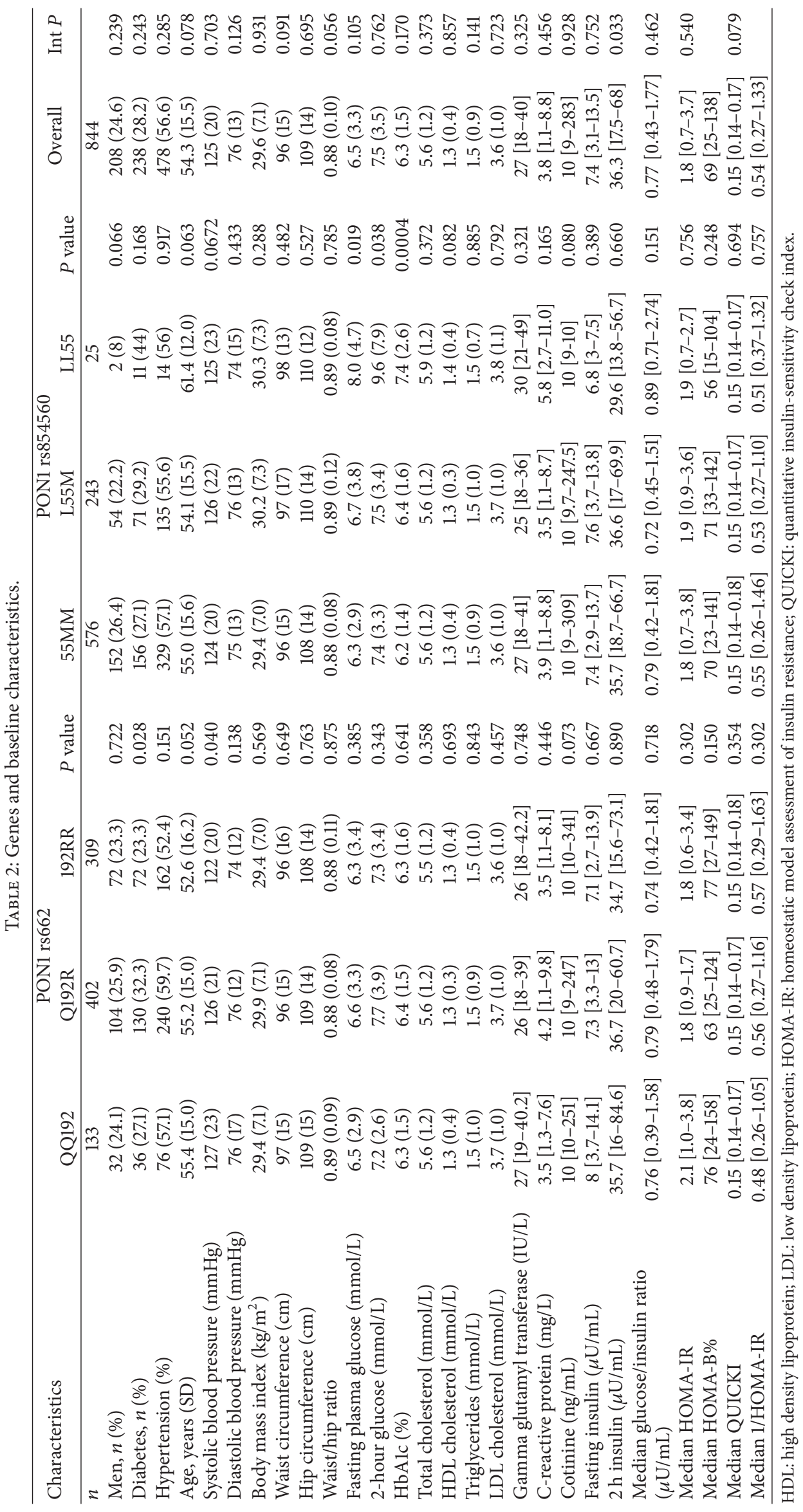




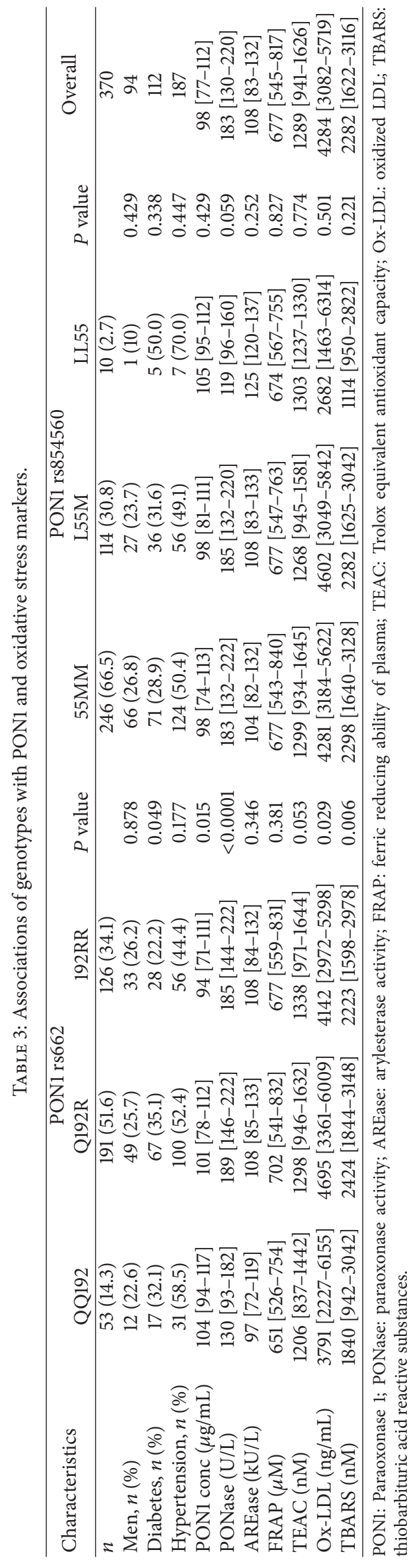




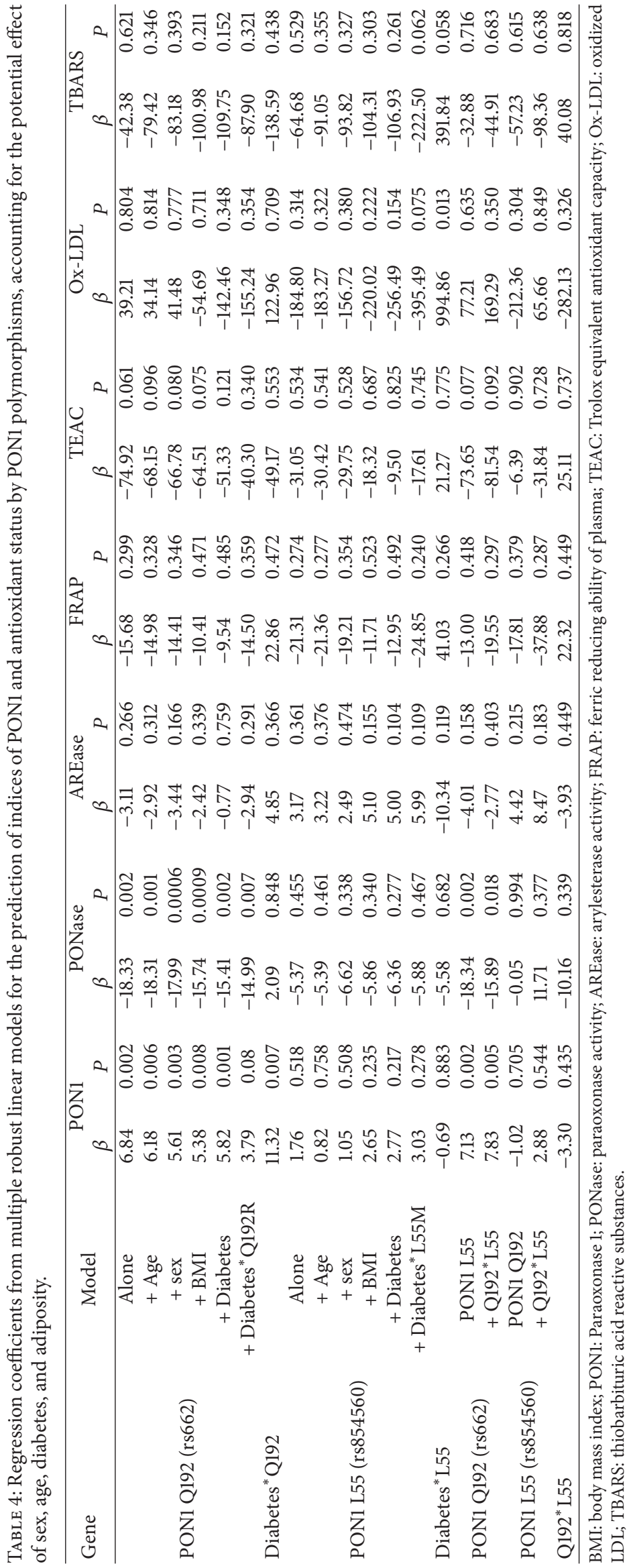


peroxidation [4]. Taken together, our results show that the $\mathrm{R}$ allele increases PON1 activity but also indicate that the PON1 QQ192 may be important in individuals with increased oxidative stress such as diabetes even though it suppresses the activity of the enzyme. Our findings, however, need to be confirmed in prospective studies with a larger sample size.

In our study we also show the predominance of PON1 $55 \mathrm{M}(82.6 \%)$ in this population, an unusual finding reported in only one other study in much lower proportion (61\%) [22]. The PON1 L55 PON1 has been associated with increased PON1 activity [45]; however, this was not apparent in this study. It is however worth noting that the distribution of indices of glycaemic control (FBG, $2 \mathrm{hr}$ glucose, and HbAlc) differs significantly across the L55M genotypes which may suggest an association with poorer glucose control and therefore glycation-enhanced oxidative stress. Although we had a relatively large population for robust linear regression studies based on the number of predictors assessed in the current study, it is likely that the low frequency of some genotypes (in PON1 L55M in particular) has affected our power for uncovering some significant associations. However, our study also has major strengths. Unlike many existing studies, in addition to PON1 polymorphisms, we measured PON1 protein levels and activity by two methods and assessed oxidative status via several methods to demonstrate the consistency of our results.

In conclusion, we have shown that the Q192R polymorphism is a determinant of both PON1 concentration and activity. This association appeared to be enhanced in subjects with diabetes, thus suggesting a need to adjust for potential genetic confounding in future PON1 studies, involving diabetic subjects.

\section{Conflict of Interests}

The authors declare that there is no conflict of interests regarding the publication of this paper.

\section{Acknowledgments}

The authors wish to thank the Bellville South Community of Cape Town, South Africa. This research was supported by grants from the University Research Fund of the Cape Peninsula University of Technology, South Africa, and South African Medical Research Council.

\section{References}

[1] M. G. von Mallinckrodt and T. L. Diepgen, "The human serum paraoxonase-polymorphism and specificity", Toxicological \& Environmental Chemistry, vol. 18, no. 2-3, pp. 79-196, 1988.

[2] H. G. Davies, R. J. Richter, M. Keifer, C. A. Broomfield, J. Sowalla, and C. E. Furlong, "The effect of the human serum paraoxonase polymorphism is reversed with diazoxon, soman and sarin," Nature Genetics, vol. 14, no. 3, pp. 334-336, 1996.

[3] M. I. Mackness, S. Arrol, and P. N. Durrrington, "Paraoxonase prevents accumulation of lipoperoxides in low-density lipoprotein," FEBS Letters, vol. 286, no. 1-2, pp. 152-154, 1991.
[4] M. Aviram, M. Rosenblat, C. L. Bisgaier, R. S. Newton, S. L. Primo-Parmo, and B. N. La Du, "Paraoxonase inhibits highdensity lipoprotein oxidation and preserves its functions: a possible peroxidative role for paraoxonase," The Journal of Clinical Investigation, vol. 101, no. 8, pp. 1581-1590, 1998.

[5] O. Rozenberg, D. M. Shih, and M. Aviram, "Paraoxonase 1 (PON1) attenuates macrophage oxidative status: Studies in PON1 transfected cells and in PON1 transgenic mice," Atherosclerosis, vol. 181, no. 1, pp. 9-18, 2005.

[6] O. Rozenberg, M. Rosenblat, R. Coleman, D. M. Shih, and M. Aviram, "Paraoxonase (PON1) deficiency is associated with increased macrophage oxidative stress: studies in PON1knockout mice," Free Radical Biology and Medicine, vol. 34, no. 6, pp. 774-784, 2003.

[7] H. Berrougui, S. Loued, and A. Khalil, "Purified human paraoxonase-1 interacts with plasma membrane lipid rafts and mediates cholesterol efflux from macrophages," Free Radical Biology and Medicine, vol. 52, no. 8, pp. 1372-1381, 2012.

[8] A. D. Watson, J. A. Berliner, S. Y. Hama et al., "Protective effect of high density lipoprotein associated paraoxonase. Inhibition of the biological activity of minimally oxidized low density lipoprotein," The Journal of Clinical Investigation, vol. 96, no. 6, pp. 2882-2891, 1995.

[9] M. Rosenblat, N. Volkova, J. Ward, and M. Aviram, "Paraoxonase 1 (PON1) inhibits monocyte-to-macrophage differentiation," Atherosclerosis, vol. 219, no. 1, pp. 49-56, 2011.

[10] Z. Ahmed, S. Babaei, G. F. Maguire et al., "Paraoxonase-1 reduces monocyte chemotaxis and adhesion to endothelial cells due to oxidation of palmitoyl, linoleoyl glycerophosphorylcholine," Cardiovascular Research, vol. 57, no. 1, pp. 225-231, 2003.

[11] M. I. Mackness, D. Harty, D. Bhatnagar et al., "Serum paraoxonase activity in familial hypercholesterolaemia and insulindependent diabetes mellitus," Atherosclerosis, vol. 86, no. 2-3, pp. 193-199, 1991.

[12] B. Mackness, P. N. Durrington, A. J. M. Boulton, D. Hine, and M. I. Mackness, "Serum paraoxonase activity in patients with type 1 diabetes compared to healthy controls," European Journal of Clinical Investigation, vol. 32, no. 4, pp. 259-264, 2002.

[13] C. Zhou, J. Cao, L. Shang et al., "Reduced paraoxonase 1 activity as a marker for severe coronary artery disease," Disease Markers, vol. 35, no. 2, pp. 97-103, 2013.

[14] R. Humbert, D. A. Adler, C. M. Disteche, C. Hassett, C. J. Omiecinski, and C. E. Furlong, "The molecular basis of the human serum paraoxonase activity polymorphism," Nature Genetics, vol. 3, no. 1, pp. 73-76, 1993.

[15] L. G. Costa, T. B. Cole, and C. E. Furlong, "Polymorphisms of paraoxonase (PON1) and their significance in clinical toxicology of organophosphates," Journal of Toxicology-Clinical Toxicology, vol. 41, no. 1, pp. 37-45, 2003.

[16] M.-C. Blatter Garin, R. W. James, P. Dussoix et al., "Paraoxonase polymorphism Met-Leu54 is associated with modified serum concentrations of the enzyme. A possible link between the paraoxonase gene and increased risk of cardiovascular disease in diabetes," Journal of Clinical Investigation, vol. 99, no. 1, pp. 62-66, 1997.

[17] S. R. Srinivasan, S. Li, W. Chen et al., "Q192R polymorphism of the paraoxanase 1 gene and its association with serum lipoprotein variables and carotid artery intima-media thickness in young adults from a biracial community: the Bogalusa Heart Study," Atherosclerosis, vol. 177, no. 1, pp. 167-174, 2004. 
[18] H. N. Luu, P. L. Kingah, K. North, E. Boerwinkle, and K. A. Volcik, "Interaction of Folate Intake and the Paraoxonase Q192R Polymorphism with Risk of Incident Coronary Heart Disease and Ischemic Stroke: the Atherosclerosis Risk in Communities Study," Annals of Epidemiology, vol. 21, no. 11, pp. 815-823, 2011.

[19] M. F. Martínez-Salazar, D. Almenares-López, S. García-Jiménez et al., "Relationship between the paraoxonase (PON1) L55M and Q192R polymorphisms and obesity in a Mexican population: a pilot study," Genes \& Nutrition, vol. 6, no. 4, pp. 361-368, 2011.

[20] W.-F. Li, M.-H. Pan, M.-C. Chung, C.-K. Ho, and H.-Y. Chuang, "Lead exposure is associated with decreased serum paraoxonase 1 (PON1) activity and genotypes," Environmental Health Perspectives, vol. 114, no. 8, pp. 1233-1236, 2006.

[21] F. A. Abessolo, M. J. Bruno, M. A. N’Negue, M. Yangou, E. Ngou-Milama, and F. Ovono, "Enzymatic and genetic polymorphisms of paraox-onase-1 in the Gabonese population: the relation to lipid parameters in patients with diabetes," Journal of Endocrinology, Metabolism and Diabetes of South Africa, vol. 17, no. 2, pp. 92-99, 2012.

[22] M. A. Ergun, E. Yurtcu, H. Demirci et al., "PON155 and 192 gene polymorphisms in type 2 diabetes mellitus patients in a Turkish population," Biochemical Genetics, vol. 49, no. 1-2, pp. 1-8, 2011.

[23] F. Sepahvand, M. Shafiei, S. M. Ghaffari, P. RahimiMoghaddam, and M. Mahmoudian, "Paraoxonase phenotype distribution in a healthy Iranian population," Basic and Clinical Pharmacology and Toxicology, vol. 101, no. 2, pp. 104-107, 2007.

[24] R. T. Erasmus, D. J. Soita, M. S. Hassan et al., "High prevalence of diabetes mellitus and metabolic syndrome in a South African coloured population: baseline data of a study in Bellville, Cape Town," South African Medical Journal, vol. 102, no. 11, pp. 841844, 2012.

[25] T. E. Matsha, M. S. Hassan, M. Kidd, and R. T. Erasmus, "The 30-year cardiovascular risk profile of South Africans with diagnosed diabetes, undiagnosed diabetes, pre-diabetes or normoglycaemia: the Bellville, South Africa pilot study," Cardiovascular Journal of Africa, vol. 23, no. 1, pp. 5-11, 2012.

[26] N. Patterson, D. C. Petersen, R. E. van der Ross et al., "Genetic structure of a unique admixed population: implications for medical research," Human Molecular Genetics, vol. 19, no. 3, Article ID ddp505, pp. 411-419, 2009.

[27] J. Chalmers, S. MacMahon, G. Mancia et al., "World Health Organization-International Society of Hypertension Guidelines for the management of hypertension. Guidelines subcommittee of the World Health Organization," Clinical and Experimental Hypertension, vol. 21, no. 5-6, pp. 1009-1060, 1999.

[28] K. G. Alberti and P. Z. Zimmet, "Definition, diagnosis and classification of diabetes mellitus and its complications. Part 1: diagnosis and classification of diabetes mellitus provisional report of a WHO consultation," Diabetic Medicine, vol. 15, no. 7, pp. 539-553, 1998.

[29] W. T. Friedewald, R. I. Levy, and D. S. Fredrickson, "Estimation of the concentration of low-density lipoprotein cholesterol in plasma, without use of the preparative ultracentrifuge," Clinical Chemistry, vol. 18, no. 6, pp. 499-502, 1972.

[30] I. F. F. Benzie and J. J. Strain, "The ferric reducing ability of plasma (FRAP) as a measure of "antioxidant power": the FRAP assay," Analytical Biochemistry, vol. 239, no. 1, pp. 70-76, 1996.

[31] R. Re, N. Pellegrini, A. Proteggente, A. Pannala, M. Yang, and C. Rice-Evans, "Antioxidant activity applying an improved ABTS radical cation decolorization assay," Free Radical Biology and Medicine, vol. 26, no. 9-10, pp. 1231-1237, 1999.
[32] R. J. Richter and C. E. Furlong, "Determination of paraoxonase (PON1) status requires more than genotyping," Pharmacogenetics, vol. 9, no. 6, pp. 745-753, 1999.

[33] R. W. Browne, S. T. Koury, S. Marion, G. Wilding, P. Muti, and M. Trevisan, "Accuracy and biological variation of human serum paraoxonase 1 activity and polymorphism (Q192R) by kinetic enzyme assay," Clinical Chemistry, vol. 53, no. 2, pp. 310317, 2007.

[34] A. M. Jentzsch, H. Bachmann, P. Fürst, and H. K. Biesalski, "Improved analysis of malondialdehyde in human body fluids," Free Radical Biology and Medicine, vol. 20, no. 2, pp. 251-256, 1996.

[35] S. A. Miller, D. D. Dykes, and H. F. Polesky, "A simple salting out procedure for extracting DNA from human nucleated cells," Nucleic Acids Research, vol. 16, no. 3, article 1215, 1988.

[36] D. L. Rainwater, S. Rutherford, T. D. Dyer et al., "Determinants of variation in human serum paraoxonase activity," Heredity, vol. 102, no. 2, pp. 147-154, 2009.

[37] I. Ahmad, R. Narang, A. Venkatraman, and N. Das, "Twoand three-locus haplotypes of the paraoxonase (PON1) gene are associated with coronary artery disease in Asian Indians," Gene, vol. 506, no. 1, pp. 242-247, 2012.

[38] Y. Imai, H. Morita, H. Kurihara et al., "Evidence for association between paraoxonase gene polymorphisms and atherosclerotic diseases," Atherosclerosis, vol. 149, no. 2, pp. 435-442, 2000.

[39] J. J. Regieli, J. W. Jukema, P. A. Doevendans et al., "Paraoxonase variants relate to 10 -year risk in coronary artery disease: impact of a high-density lipoprotein-bound antioxidant in secondary prevention," Journal of the American College of Cardiology, vol. 54, no. 14, pp. 1238-1245, 2009.

[40] T. Bhattacharyya, S. J. Nicholls, E. J. Topol et al., "Relationship of paraoxonase 1 (PON1) gene polymorphisms and functional activity with systemic oxidative stress and cardiovascular risk," The Journal of the American Medical Association, vol. 299, no. 11, pp. 1265-1276, 2008.

[41] D. M. Shih and A. J. Lusis, "The roles of PON1 and PON2 in cardiovascular disease and innate immunity," Current Opinion in Lipidology, vol. 20, no. 4, pp. 288-292, 2009.

[42] W. H. W. Tang, J. Hartiala, Y. Fan et al., "Clinical and genetic association of serum paraoxonase and arylesterase activities with cardiovascular risk," Arteriosclerosis, Thrombosis, and Vascular Biology, vol. 32, no. 11, pp. 2803-2812, 2012.

[43] L. Gaidukov, M. Rosenblat, M. Aviram, and D. S. Tawfik, "The 192R/Q polymorphs of serum paraoxonase PON1 differ in HDL binding, lipolactonase stimulation, and cholesterol efflux," Journal of Lipid Research, vol. 47, no. 11, pp. 2492-2502, 2006.

[44] B. Mackness, P. N. Durrington, and M. I. Mackness, "Polymorphisms of paraoxonase genes and low-density lipoprotein lipid peroxidation," The Lancet, vol. 353, no. 9151, pp. 468-469, 1999.

[45] D. Altuner, I. Ates, S. H. Suzen, G. V. Koc, Y. Aral, and A. Karakaya, "The relationship of PON1 QR 192 and LM 55 polymorphisms with serum paraoxonase activities of Turkish diabetic patients," Toxicology and Industrial Health, vol. 27, no. 10, pp. 873-878, 2011. 


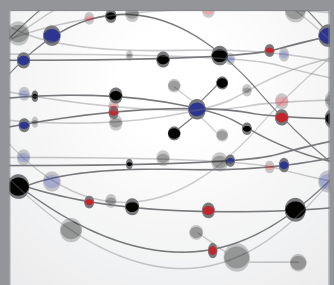

The Scientific World Journal
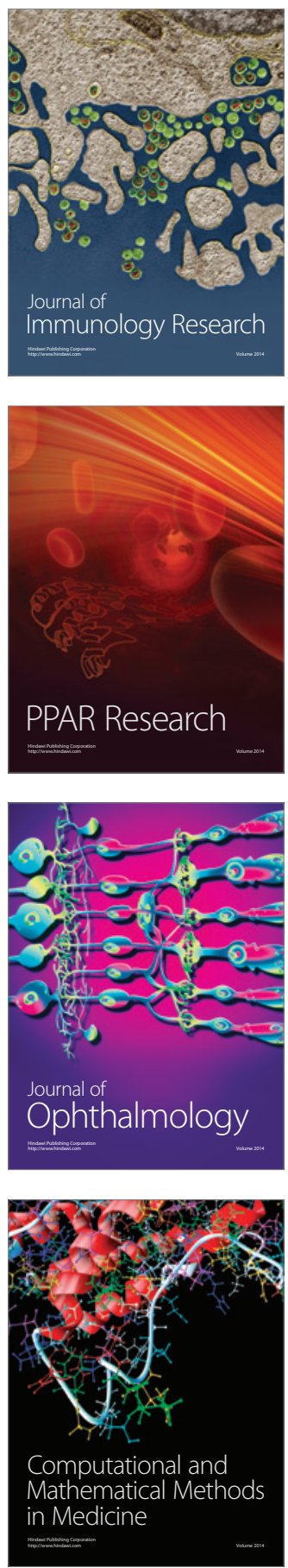

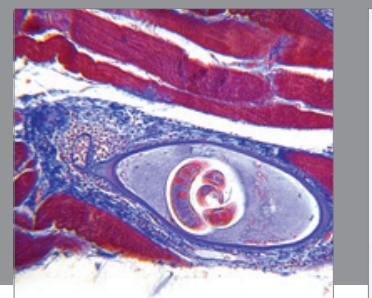

Gastroenterology

Research and Practice
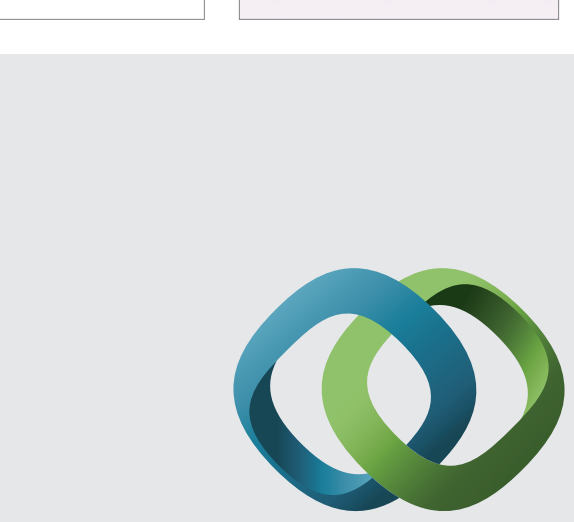

\section{Hindawi}

Submit your manuscripts at

http://www.hindawi.com
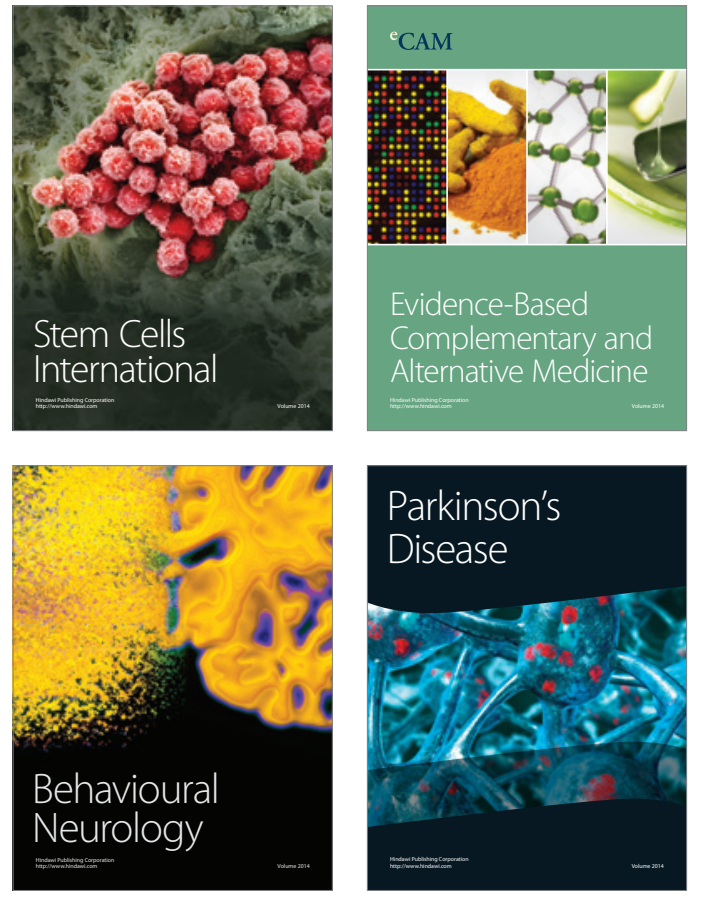
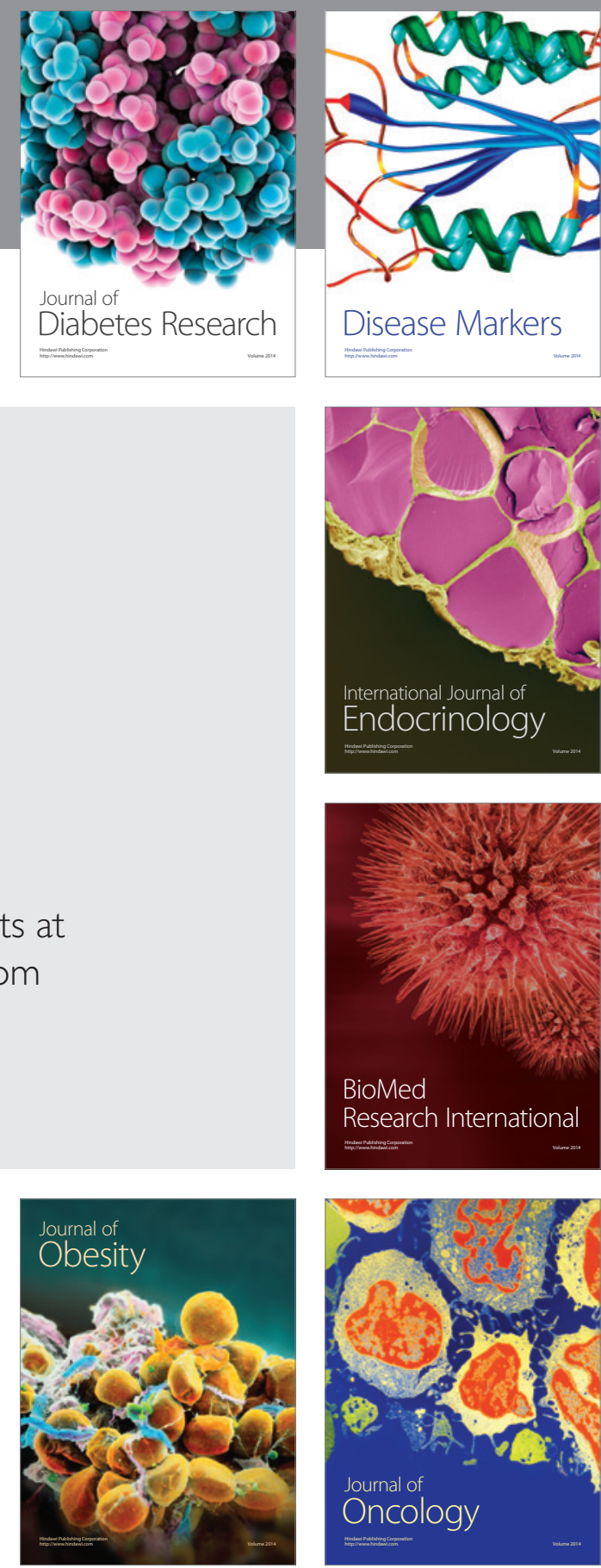

Disease Markers
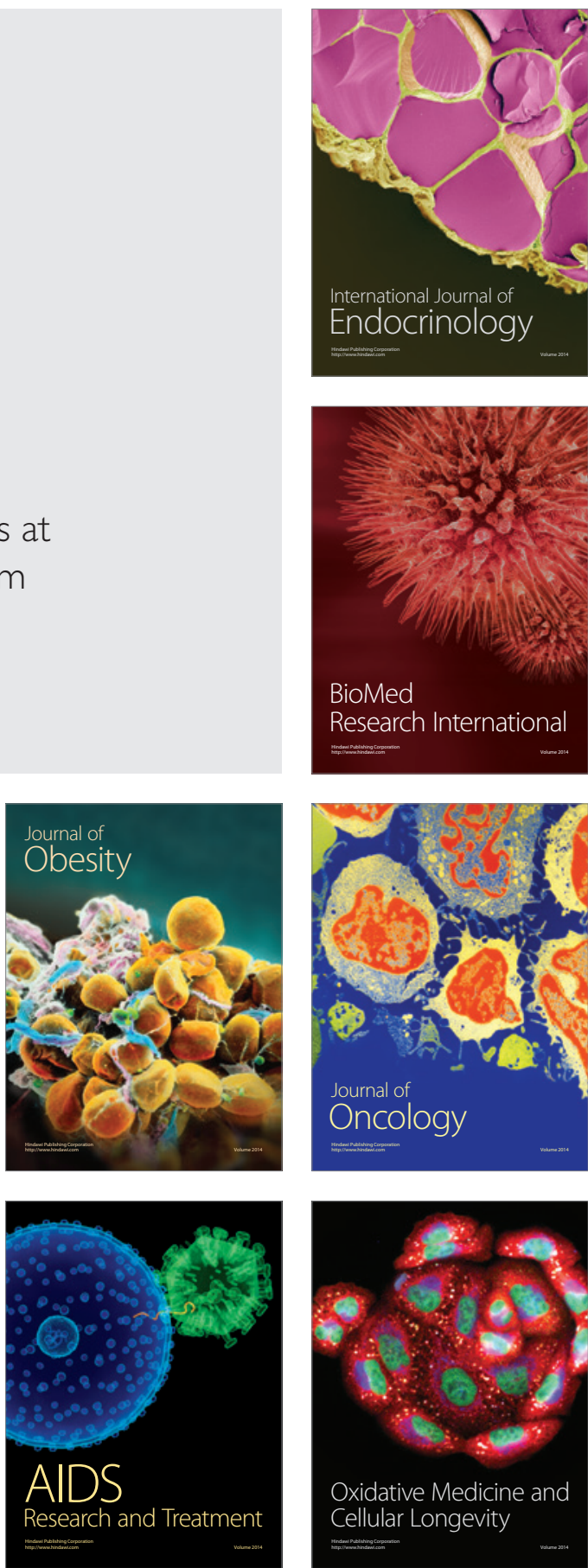